\title{
Registration of brain CT images to an MRI template for the purpose of lesion-symptom mapping ${ }^{\star}$
}

\author{
Hugo J. Kuijf, J. Matthijs Biesbroek, Max A. Viergever, Geert Jan Biessels, \\ and Koen L. Vincken \\ University Medical Center Utrecht, the Netherlands
}

\begin{abstract}
Lesion-symptom mapping is a valuable tool for exploring the relation between brain structure and function. In order to perform lesion-symptom mapping, lesion delineations made on different brain CT images need to be transformed to a standardized coordinate system. The preferred choice for this is the MNI152 template image that is based on T1-weighted MR images. This requires a multi-modal registration procedure to transform lesion delineations for each CT image to the MNI152 template image. A two-step registration procedure was implemented, using lesion-masking and contrast stretching to correctly align the soft tissue of the CT image to the MNI152 template image. The results were used to transform the lesion delineations to the template. The quality of the registration was assessed by an expert human observer. Of the 86 CT images, the registration was highly successful in 71 cases (83\%). Slight manual adjustments of the lesion delineations in the standard coordinate system were required to make unsuccessful cases suitable for a lesion-symptom mapping study.
\end{abstract}

\section{Introduction}

Acute ischemic stroke frequently causes cognitive deficits. [1] Besides the volume of lesions, the exact location of lesions is an important factor in explaining the variance in post-stroke cognitive performance. [1] The study of these patients with acquired brain damage is a valuable tool for exploring the relationship between brain structure and function. Lesion-symptom mapping studies have provided valuable insights in neuroanatomical correlates of various cognitive functions. [2]

Traditional approaches to lesion-symptom mapping include comparing the performance of groups of patients with and without lesions at one or more predefined locations. [2,3] Recently, more sophisticated methods have emerged, such as voxel-based lesion-symptom mapping (VLSM). [3,4] A major advantage of VLSM over traditional approaches is that it allows for assumption-free calculation of associations between brain injury and behavioral performance at each individual location (i.e. voxel) in the image.

* The final publication is available at Springer via http://dx.doi.org/10.1007/978-3319-02126-3_12. 
A prerequisite for performing voxel-wise analyses is that the lesions have to be segmented and registered to a standard space. Segmentation of the lesions is done by delineating the location of lesions manually or (semi-)automatically on structural imaging (usually CT or MRI T1- or T2-weighted sequences), resulting in a lesion mask for each subject. Next, all lesion masks are transformed into a standardized coordinate system. Each voxel in this standardized coordinate system contains information regarding the presence or absence of a lesion at that specific location for each subject. Finally, a VLSM approach is used to investigate the relation between the occurrence of a lesion at a specific location and cognitive performance.

The MNI152 standard-space MRI T1-weighted average structural template image (shortened as: MNI152 template) is often used as the standardized coordinate system that all lesion masks are transformed to. $[5,6]$ This MNI152 template is derived from 152 individual images, as described by Fonov et al. For this template, a number of anatomical atlases are available, making it a popular choice when performing lesion-symptom mapping. [7]

Ischemic brain lesions are often imaged using CT-because of higher availability and shorter acquisition time - whereas the MNI152 template is based on MR images. This complicates the registration procedure. A common solution is to acquire a T1-weighted MR image of each subject and perform a two-step registration. Hereby the $\mathrm{CT}$ image is registered to the T1-weighted image and the T1-weighted image to the MNI152 template. Both transformations are combined to transform the $\mathrm{CT}$ image directly to the standard coordinate space. If T1-weighted images are not available, which is the case by imaging of acute ischemic lesions, the CT image has to be registered to the MNI152 template directly. This is more complicated, because the registration procedure has to deal with both the difference in modality and shape of the head.

In literature, some methods are described to perform this inter-subject registration between $\mathrm{CT}$ and MR (template) images. $[8,9]$ The general consensus is that a deformable registration optimizing a mutual information metric is required. However, details of the practical implementation of this approach are not always described. Especially for the purpose of lesion-symptom mapping studies, where the presence of brain lesions and the low soft tissue contrast in the CT images complicates the registration. A recent publication by Gao et al. describes a procedure for registering brain CT images to an MRI template, by including the mid-sagittal plane to constrain the deformable registration. [10] However, the presence of brain lesions is not reported and the results were only evaluated for the total brain volume, disregarding the difficulties of registering the soft tissue within the brain.

The goal of the present study is to develop a method to transform lesion masks to a standardized space for the purpose of lesion-symptom mapping, by registration of CT images directly to the MNI152 template. The proposed method consists of two steps: an affine registration to globally align the CT image with the MNI152 template and a deformable registration to locally match 
the template. The resulting transform is applied to the delineated lesions and the quality of the transformed lesion masks is assessed.

\section{Methods and Materials}

\subsection{Participants \& CT}

From a prospectively collected database of patients who were admitted between November 2005 and December 2012 with ischemic stroke to the University Medical Center Utrecht, we included 86 patients. Patients fulfilled the following inclusion criteria: (1) first-ever ischemic stroke; (2) an infarction on follow-up CT; (3) no infarcts from earlier date. Because the data were gathered in order to study the relation between lesion location and cognitive performance, patients with known pre-existent impaired cognition were also excluded.

All CT scans were performed in the setting of standard clinical care and were acquired using a Philips Mx8000 16, Brilliance 64, or Brilliance iCT 256 CT scanner.

Lesion locations were manually delineated on the CT images by an experienced observer, with an in-house developed tool based on MeVisLab (MeVis Medical Solutions AG, Bremen, Germany [11]).

\subsection{Affine registration}

All registrations, both affine and deformable, were performed with the elastix toolbox. [12] The used MNI152 template has a voxel size of $1.0 \mathrm{~mm}^{3}$. To globally align the CT image with the MNI152 template, an affine registration considering only the bone was performed.

The bone was extracted from the CT images: intensity values below $500 \mathrm{HU}$ were set to zero, intensity values equal to or above $500 \mathrm{HU}$ were untouched. The MNI152 template was modified to extract the bone. First, the template image intensities were inverted to produce high intensity values at the location of the bone. Next, the image was thresholded to extract the bone and a mask was applied to suppress the background. An example is shown in Figure 1. Note that the ventricles are visible in the bone-extracted MNI152 template image, because of their low intensities. This does not influence the registration, since there are no intensities to match in the bone-extracted CT image.

An affine registration was used to transform the bone-extracted CT image to the bone-extracted MNI152 template, following the recommended settings of elastix: a multi-resolution approach with four resolutions, the Advanced Mattes Mutual Information metric, and the Adaptive Stochastic Gradient Descent optimizer. [13-15]

\subsection{Deformable registration}

Following the affine registration to align the bone-extracted CT and MNI152 images, a deformable registration was used to align the soft tissue of the brain 


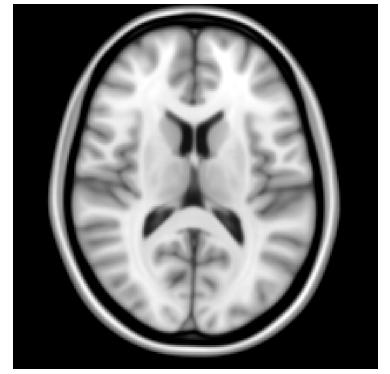

(a)

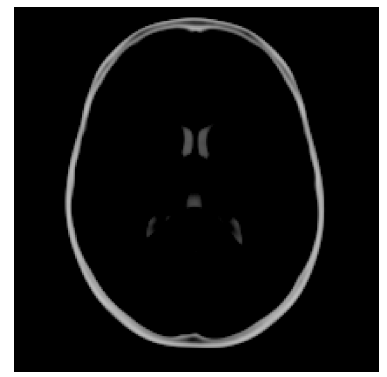

(d)

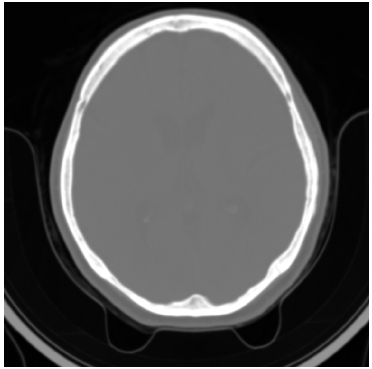

(b)

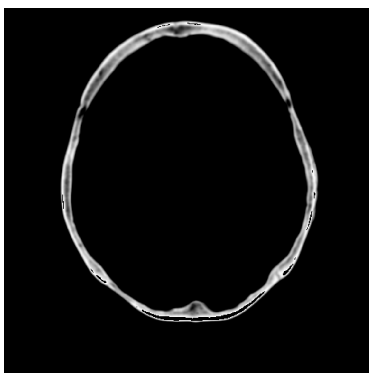

(e)

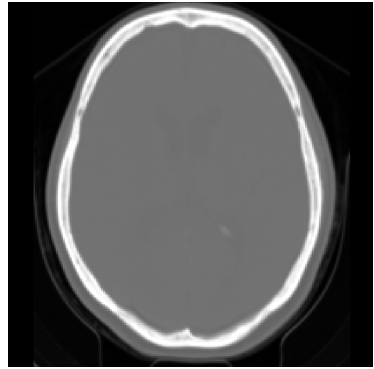

(c)

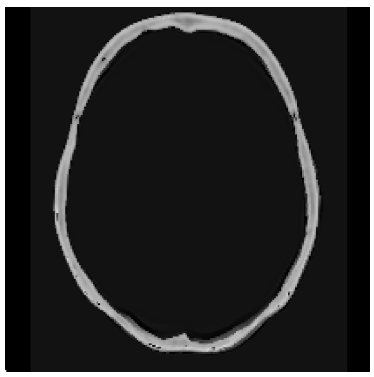

(f)

Fig. 1. Left: (a) the MNI152 template image and (d) the bone-extracted image. Middle: (b) an example CT image and (e) the bone-extracted image. The image in (e) was transformed to (d) with an affine registration. Right: transformed CT image after registration. 
with the template. A B-spline with its control points placed in a regular 3D grid, spaced $10 \mathrm{~mm}$ apart, was used to deform the CT image. [16,17] The Advanced Mattes Mutual Information metric was optimized with a standard gradient descent optimizer. [18] The previously computed affine transform and the B-spline transform were composed together, to avoid repeated interpolations. A lesionmasking approach was used to prevent a bias in the transformation caused by the presence of the lesion, by ignoring the manually delineated lesion area in the computation of the metric. [19]

However, the lack of sufficient contrast in soft tissue of the brain on CT images makes it challenging to directly transform such images to the MNI152 template. The features that need to be aligned with the MNI152 templatethe gray matter, white matter, and cerebrospinal fluid - all have intensity values between $0 \mathrm{HU}$ and $50 \mathrm{HU}$. The used Advanced Mattes Mutual Information metric computes a joint histogram of the intensity values of the CT image and MNI152 template. The number of bins of this joint histogram is set to 32 bins (elastix default value). This results in a bin size of approximately $90 \mathrm{HU}$, rendering it impossible to make a meaningful deformation of the CT image: the little contrast that was present in the soft tissue vanished into a single bin. This is illustrated in the top row of Figure 2. The effect of this can be seen in Figure 3(c), where the soft tissue after deformable registration is identical to the soft tissue after affine registration in Figure 3(b).

To overcome this, contrast stretching by means of histogram equalization and the removal of (outlier) intensities below the 5th and above the 95th percentile was applied. Furthermore, the number of bins of the joint histogram was increased to 64 bins. By doing this, the intensities of the gray matter, white matter, and cerebrospinal fluid were distributed over approximately 15 bins in the joint histogram, as can be seen in Figure 2. This enabled the registration procedure to compute a meaningful deformation of the CT image to the MNI152 template, as can be seen in Figure 3.

\subsection{Experiments}

The described procedure was used to transform the CT images and the lesion delineations of the 86 participants to the MNI152 template. Since the proposed method was developed for the purpose of lesion-symptom mapping, a correct positioning of the lesions was the main goal. An experienced human observer visually assessed the quality of the transformation of the lesion delineations and determined if the quality was sufficient for VLSM.

\section{Results}

Of the $86 \mathrm{CT}$ images, the registration was successful in 71 cases (83\%). Some example results are shown in Figure 4.

In eight of the unsuccessful cases there was a misalignment of the tentorium cerebelli (separates cerebellum from cerebral hemispheres). An example is shown 


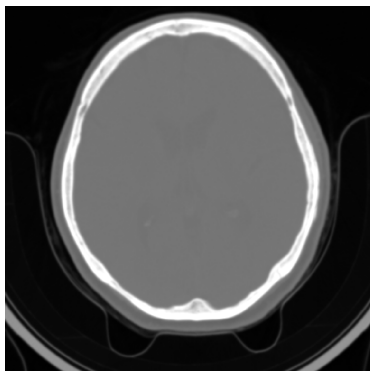

(a)

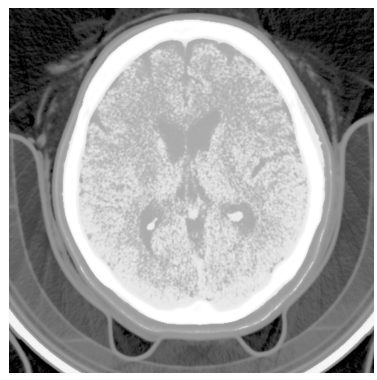

(c)

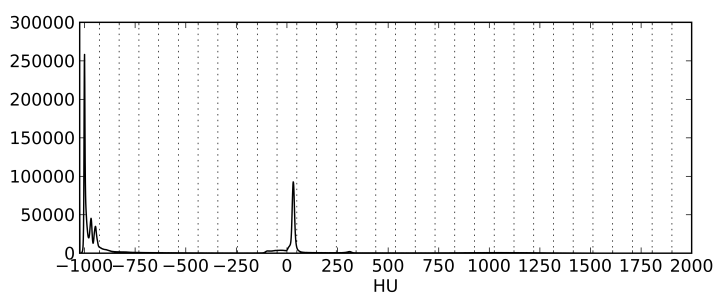

(b)

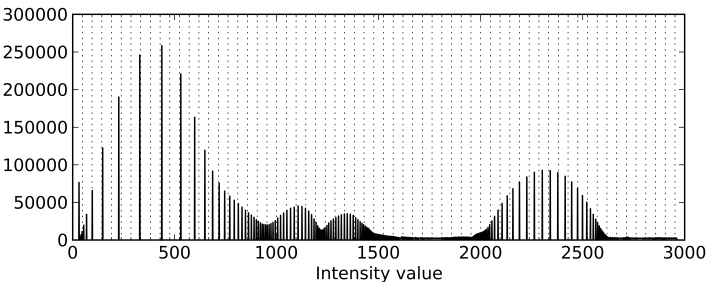

(d)

Fig. 2. Top row: original CT image with its histogram of intensities. The peak around $25 \mathrm{HU}$ represents the soft tissue; the dotted vertical lines are the histogram bins used by the metric. Bottom row: contrast enhanced CT image with its histogram. The soft tissue now has intensity values between 2000 and 2500, which are spread over more bins in the histogram. The number of histogram bins has been increased as well (dotted lines). 


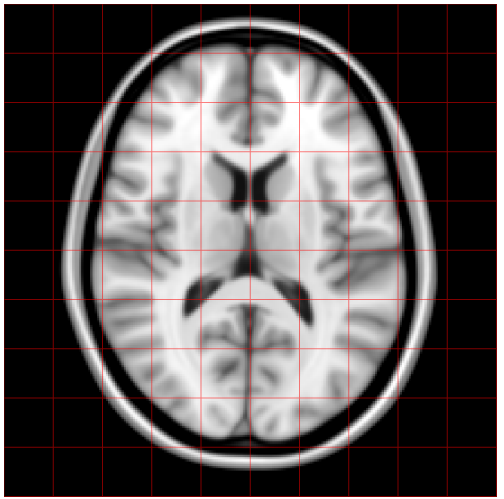

(a)

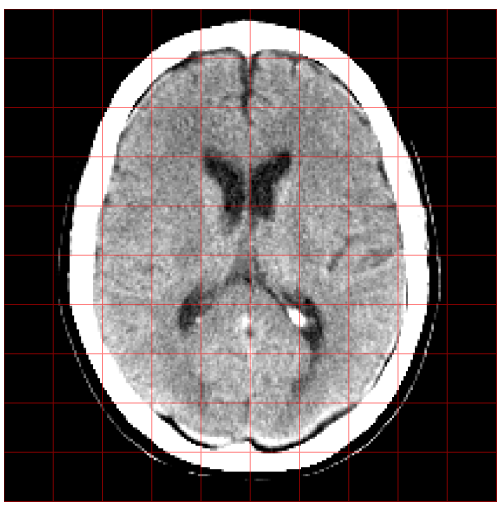

(c)

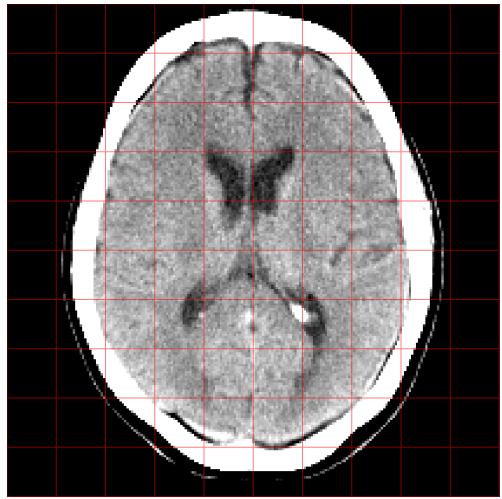

(b)

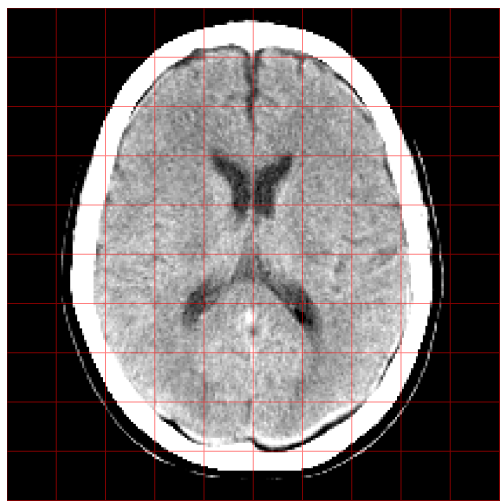

(d)

Fig. 3. The effect of contrast stretching on the deformable registration. Note that for viewing purposes only, the contrast settings have been altered to highlight the soft tissue and a reference grid is included. (a) The MNI152 template. (b) The CT image after affine registration. (c) The CT image after deformable registration without contrast stretching. (d) The CT image after deformable registration with contrast stretching. 


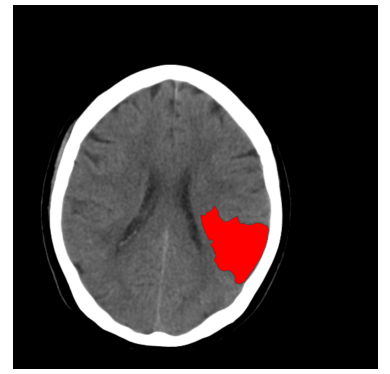

(a)

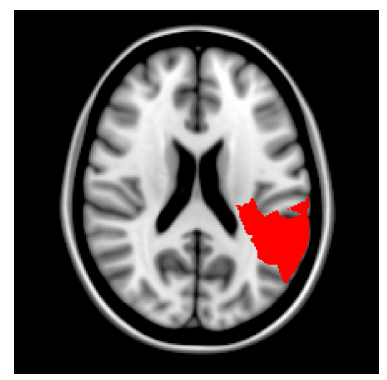

(d)

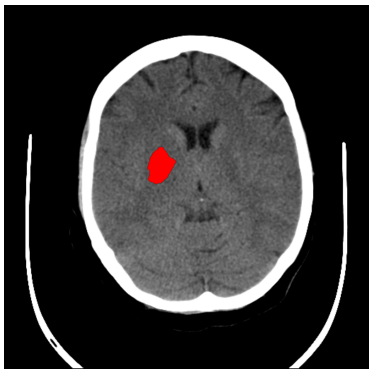

(b)

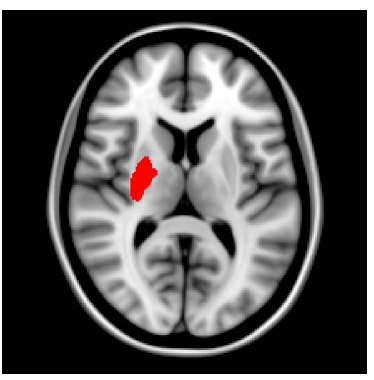

(e)

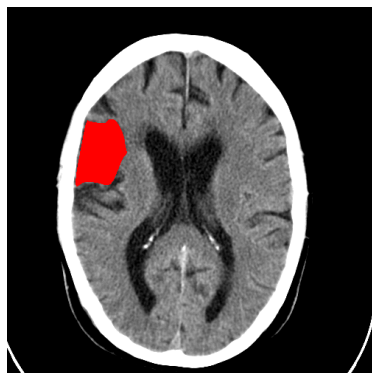

(c)

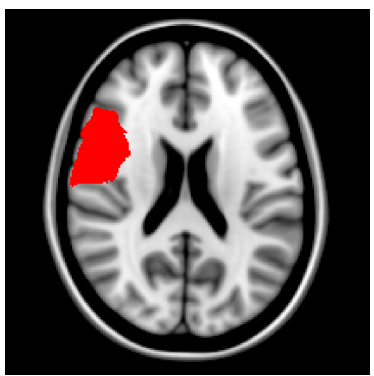

(f)

Fig. 4. Top row: original CT images with the lesion delineation overlaid in red. Note that the viewing settings have been altered to highlight the soft tissue. Bottom row: lesion delineations transformed to the MNI152 template image. 
in the top row of Figure 5. In six unsuccessful cases there was a misalignment of the ventricles, caused by either edema with midline shift (four cases, example in bottom row of Figure 5) or atrophy with enlargement of the ventricular system (two cases). In one unsuccessful case there was no evident cause. All misalignments were resolved by slight manual adjustments of the lesion delineations in the standard space, as can be seen in Figures 5(c) and (f).

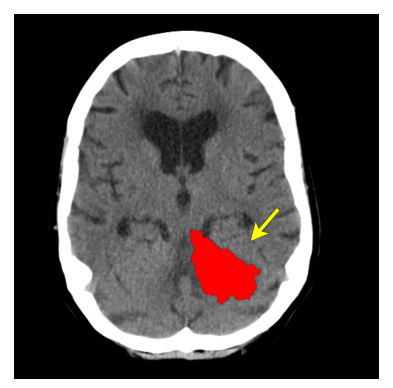

(a)

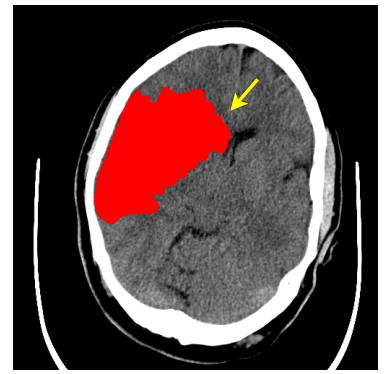

(d)

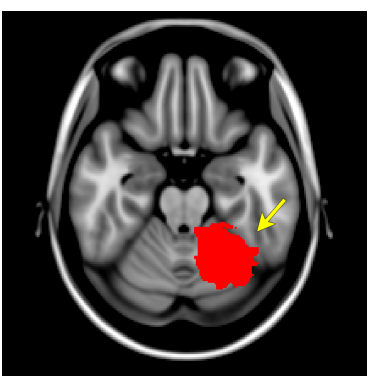

(b)

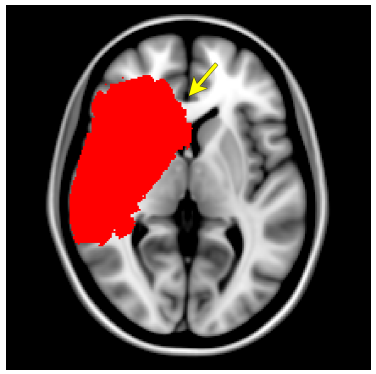

(e)

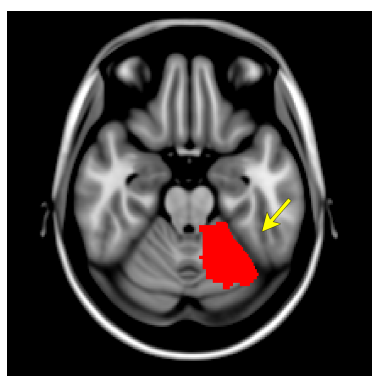

(c)

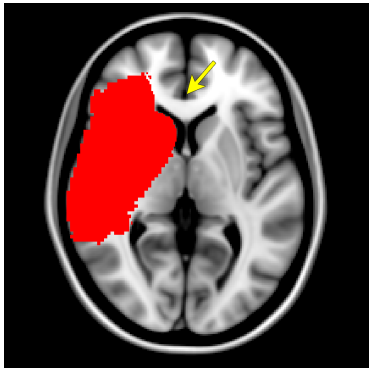

(f)

Fig. 5. Top row: (a) original CT with lesion delineation in red, (b) transformed lesion delineation on MNI152 template image is incorrect around the tentorium cerebelli, (c) manually adjusted lesion delineation. Bottom row: (d) original CT with lesion delineation in red, (e) transformed lesion delineation on MNI152 template image is incorrect because of edema with midline shift, (f) manually adjusted lesion delineation.

\section{Discussion}

The proposed registration procedure to transform lesion delineations from CT images to the MNI152 template image proved to be highly successful. In $83 \%$ of the cases, the lesion delineations were correctly transformed and suitable for usage in a lesion-symptom mapping study. Slight manual adjustments of the transformed lesion delineations were needed in the remaining cases.

Performing an initial affine registration with bone-extracted images was required in order to correctly align the CT image with the MNI152 template. 
Otherwise, there is a risk that the optimization procedure will converge into a local optimum when only using a deformable registration. The bone of the CT image might then be aligned with the gray matter on the MNI152 template, as there are strong edges at those locations. The presence of the ventricles in the bone-extracted MNI152 template (see Figure 1(d)) did not influence the affine registration, since there are no corresponding intensities at that location in the bone-extracted CT image.

The deformable registration used a contrast stretched CT image to perform the registration with the MNI152 template. By applying a simple contrast stretching, the CT image intensities were spread over multiple bins in the joint histogram of the used metric. This allowed the registration procedure to correctly align the gray matter, white matter, and cerebrospinal fluid.

Unfortunately, this issue is not addressed by the publication of Gao et al. [10] In this publication, Dice's coefficient and the Hausdorff distance are reported in the results, but only for the total brain volume. The accuracy of the alignment of structures within the brain tissue (for example cortical gray matter, white matter, basal ganglia, and thalamus) are not evaluated. When performing lesionsymptom mapping, accurate alignment of these structures is essential.

The final goal of the proposed registration procedure was to transform lesion delineations, in order to perform VLSM. Therefore, the quality of the resulting transformations was assessed by an experienced observer with this goal in mind. Lesion delineations on the original CT images were visually compared to the transformed lesion delineations on the MNI152 template image. In the case that slight manual adjustments were needed to correct misalignments, the registration was deemed unsuccessful. However, as can be seen in Figure 5, large portions of the lesion delineations were correctly transformed in all the unsuccessful cases. In eight unsuccessful cases there was a misalignment of the tentorium cerebelli, a structure that is hardly visible on the CT images and therefore difficult to transform correctly. However, lesions generally do not cross the tentorium cerebelli. Manual adjustments in the standard coordinate space are thus easily made by erasing the inaccurate parts. In the unsuccessful cases caused by edema with midline shift, one of the lateral ventricles is suppressed by the lesion. Because of the lesion masking approach and the absence of a lateral ventricle, the deformation field is interpolated at these areas, since there are no features to compute the metric. This results in an enlarged lesion in the standard coordinate space, which is easily corrected by erasing the parts that overlap the lateral ventricles. Inclusion of the mid-sagittal plane, as proposed by Gao et al., is unlikely to solve this problem, because computation of the mid-sagittal plane is inaccurate in the case of midline shift. The inclusion of a (curved) mid-sagittal surface [20] constraint might prevent the lesion from being transformed into the wrong hemisphere, but the absence of a lateral ventricle is the largest problem in these cases. 


\section{Conclusion}

In this work, we proposed a procedure for the registration of $\mathrm{CT}$ images to the MNI152 template image for the purpose of lesion-symptom mapping. Lesion delineations on the CT images were successfully transformed to the MNI152 template image. Slight manual adjustments of the transformed lesion delineations were needed in a limited number of cases.

\section{References}

1. Gorelick, P.B., Scuteri, A., Black, S.E., DeCarli, C., Greenberg, S.M., Iadecola, C., Launer, L.J., Laurent, S., Lopez, O.L., Nyenhuis, D., Petersen, R.C., Schneider, J.A., Tzourio, C., Arnett, D.K., Bennett, D.A., Chui, H.C., Higashida, R.T., Lindquist, R., Nilsson, P.M., Roman, G.C., Sellke, F.W., Seshadri, S.: Vascular contributions to cognitive impairment and dementia: A statement for healthcare professionals from the american heart association/american stroke association. Stroke 42(9) (2011) 2672-2713

2. Rorden, C., Karnath, H.O.: Using human brain lesions to infer function: a relic from a past era in the fmri age? Nature Reviews Neuroscience 5(10) (2004) 812-819

3. Biesbroek, J.M., Kuijf, H.J., van der Graaf, Y., Vincken, K.L., Postma, A., Mali, W.P.T.M., Biessels, G.J., Geerlings, M.I., on behalf of the SMART Study Group: Association between subcortical vascular lesion location and cognition: A voxelbased and tract-based lesion-symptom mapping study. the smart-mr study. PLoS ONE 8(4) (04 2013) e60541

4. Rorden, C., Karnath, H.O., Bonilha, L.: Improving lesion-symptom mapping. Journal of Cognitive Neuroscience 19(7) (2007) 1081-1088

5. Fonov, V., Evans, A., McKinstry, R., Almli, C., Collins, D.: Unbiased nonlinear average age-appropriate brain templates from birth to adulthood. NeuroImage 47, Supplement 1 (2009) S102 Organization for Human Brain Mapping 2009 Annual Meeting.

6. Fonov, V., Evans, A.C., Botteron, K., Almli, C.R., McKinstry, R.C., Collins, D.L.: Unbiased average age-appropriate atlases for pediatric studies. NeuroImage 54(1) (2011) $313-327$

7. Evans, A.C., Janke, A.L., Collins, D.L., Baillet, S.: Brain templates and atlases. NeuroImage 62(2) (2012) 911-922

8. Maintz, J.B.A., Viergever, M.A.: A survey of medical image registration. Medical Image Analysis 2(1) (1998) $1-36$

9. Pluim, J.P.W., Maintz, J.B.A., Viergever, M.A.: Mutual-information-based registration of medical images: a survey. IEEE Transactions on Medical Imaging 22(8) (2003) 986-1004

10. Gao, A., Chen, M., Hu, Q.: Non-rigid registration between brain ct images and mri brain atlas by combining grayscale information, point correspondence on the midsaggital plane and brain surface matching. In: Proceedings of the 2nd International Conference on Computer Science and Electronics Engineering. Advances in Intelligent Systems Research (2013) 222-225

11. Ritter, F., Boskamp, T., Homeyer, A., Laue, H., Schwier, M., Link, F., Peitgen, H.O.: Medical image analysis: A visual approach. IEEE Pulse 2(6) (Nov.-Dec. 2011) 60-70 
12. Klein, S., Staring, M., Murphy, K., Viergever, M.A., Pluim, J.P.W.: elastix: A toolbox for intensity-based medical image registration. IEEE Transactions on Medical Imaging 29(1) (jan. 2010) 196 -205

13. Thevenaz, P., Unser, M.: Optimization of mutual information for multiresolution image registration. IEEE Transactions on Image Processing 9(12) (2000) 20832099

14. Klein, S., Pluim, J.P.W., Staring, M., Viergever, M.A.: Adaptive stochastic gradient descent optimisation for image registration. International Journal of Computer Vision 81(3) (March 2009) 227-239

15. Klein, S., Staring, M.: elastix: the manual. 4.6 edn. (2012)

16. Rueckert, D., Sonoda, L.I., Hayes, C., Hill, D.L.G., Leach, M.O., Hawkes, D.: Nonrigid registration using free-form deformations: application to breast mr images. IEEE Transactions on Medical Imaging 18(8) (1999) 712-721

17. Unser, M.: Splines: a perfect fit for signal and image processing. IEEE Signal Processing Magazine 16(6) (1999) 22-38

18. Klein, S., Staring, M., Pluim, J.P.W.: Evaluation of optimization methods for nonrigid medical image registration using mutual information and b-splines. IEEE Transactions on Image Processing 16(12) (2007) 2879-2890

19. Brett, M., Leff, A.P., Rorden, C., Ashburner, J.: Spatial normalization of brain images with focal lesions using cost function masking. NeuroImage 14(2) (2001) $486-500$

20. Kuijf, H.J., Viergever, M.A., Vincken, K.L.: Automatic extraction of the curved midsagittal brain surface on $\mathrm{mr}$ images. In: Medical Computer Vision. Recognition Techniques and Applications in Medical Imaging. Volume 7766 of Lecture Notes in Computer Science. Springer Berlin Heidelberg (2013) 225-232 